\title{
Simulation of an Airy Beam with Optical Vortex under Fractional Fourier Transforms
}

\author{
Forouzan. Habibi ${ }^{a}$ and Mohammad. Moradi*, a, b \\ aDepartment of Physics, University of Shahrekord, Shahrekord, Iran \\ bPhotonic Research Group, University of Shahrekord, Shahrekord, Iran \\ *Corresponding Author: moradi@sci.sku.ac.ir
}

Received: Jun. 3, 2018, Revised: Sep. 1, 2018, Accepted: Oct. 30, 2018, Available Online: Dec. 27, 2019

DOI: 10.29252/ijop.13.2.215

\begin{abstract}
First, this study obtained the fields of an Airy beam (AiB) with optical vortex (OV) for a Fourier transform (FT) system and a fractional Fourier transform (fractional FT) system; thereafter, their intensity and phase patterns were simulated numerically. The splitting on each line of the phase pattern indicates the position of an $\mathrm{OV}$. The results show that the OV position will change when the power of the fractional FT (p) changes. Moreover, the uniformity of the spot beam disappears for the beam with OV. Further, the characteristics of an AiB such as number, width, height, uniformity of the spot beam and the effective beam size will change when there is a change in the values of $p$ and $z$.
\end{abstract}

KEYWORDS: Fractional FT, optical vortex, Airy beam.

\section{INTRODUCTION}

AiB has numerous features and they were considered in the last decade. Although the concept of the Airy wave package dates back 30 years [1], the counterpart in optics was only demonstrated recently. In 2007, an AiB was produced experimentally [2]. In 2008, vector evolution, angular momentum and $\mathrm{AiB}$ phase behavior were investigated [3, 4]. Several methods, including a method of the Wigner distribution function for describing the features of an AiB have been presented [5]. Many investigations have been made in this regard, such as the propagation of the $\mathrm{AiB}$ in free space, in the nonlinear medium [6], in the turbulence [7] and of the misaligned optical system [8]. The propagation of an $\mathrm{AiB}$ with OV $[9,10]$ and propagation of an Airy-
Gaussian beam with OV in the chiral medium are investigated [11]. In 2012, the researchers by studying the AiB showed that the scope of the analysis of the $\mathrm{AiB}$ characteristic for its propagation through the fractional FT system is more than the FT system. In this case, assuming that a piece of graded-index fiber with proper length $\mathrm{L}$ is required for performing a FT of an input image. If the graded-index fiber is cut into pieces, a piece of length $p l(p<1)$ just performs the fractional FT of the input image [12]. The research in the $\mathrm{AiB}$ with OV at the fractional FT has not been reported. Previously, a study was conducted on the $\mathrm{AiB}$ that was propagated through the fractional FT system. Now, we are inspired by the work of the researchers [12], studying the $\mathrm{AiB}$ with $\mathrm{OV}$ that propagates through the fractional FT system and then simulate its intensity and phase pattern numerically. We want to know, how the AiB with OV behaves by changing the order of the fractional FT and distance in the optical system.

\section{THEORETICAL CONCEPT}

\section{A. FT System}

The field of an AiB is defined with OV as [9]:

$$
\begin{aligned}
E(x, y, z=0)= & A i\left(x / x_{0}\right) \exp \left(a x / x_{0}\right) A i\left(y / y_{0}\right) \times \\
& \exp \left(a y / y_{0}\right)\left(\left(x-x_{d}\right)+i\left(y-y_{d}\right)\right)^{l} .
\end{aligned}
$$

where the parameters $x_{0}$ and $y_{0}$ are transverse scales, and $a$ is the truncation factor exponential; which characterizes beams, $l$ is the topological charge of the vortex. For simplicity, we choose the unit topological 
charge and the OV core center is in the coordinates $\left(x_{d}, y_{d}\right)$. The Fresnel integral can be used to calculate the field of the beam at distance $z$ in the FT system [13]. On substituting Eq. (1) into (2), the field of the AiB with OV can be obtained, after it propagates a distance $z$.

$$
\begin{aligned}
& E(x, y, z)=\frac{i k}{2 \pi z} \times \\
& \iint d x_{1} d y_{1} \exp \left\{\frac{i k}{2 z}\left[\left(x_{1}-x\right)^{2}+\left(y_{1}-y\right)^{2}\right]\right\} \times \\
& E\left(x_{1}, y_{1}, z=0\right)=\frac{i k}{2 \pi z} \iint d x_{1} d y_{1} \exp \left\{\frac { i k } { 2 z } \left[\left(x_{1}-x\right)^{2}+\right.\right. \\
& \left.\left.\left(y_{1}-y\right)^{2}\right]\right\} A i\left(\frac{x_{1}}{x_{0}}\right) A i\left(\frac{y_{1}}{y_{0}}\right) \exp \left(\frac{a x_{1}}{x_{0}}+\frac{a y_{1}}{y_{0}}\right) \times \\
& {\left[\left(x_{1}-x_{d}\right)+i\left(y_{1}-y_{d}\right)\right]}
\end{aligned}
$$

and

$$
\begin{aligned}
& E(x, y, z)=\frac{i k}{2 \pi z} \exp \left(\frac{a x}{x_{0}}-\frac{i a^{2} z}{2 k x_{0}^{2}}+\frac{a y}{y_{0}}-\frac{i a^{2} z}{2 k y_{0}^{2}}\right) \times \\
& \left\{\int d x_{1} \exp \left(\frac{i k}{2 z}\left[\left(x_{1}-\left(x-\frac{i a z}{x_{0} k}\right)\right)^{2}\right]\right) A i\left(\frac{x_{1}}{x_{0}}\right)\left(x_{1}-x_{d}\right)\right. \\
& \times \int d y_{1} \exp \left(\frac{i k}{2 z}\left[y_{1}-\left(y-\frac{i a z}{y_{0} k}\right)\right]^{2}\right) A i\left(\frac{y_{1}}{y_{0}}\right)+ \\
& \int d x_{1} \exp \left(\frac{i k}{2 z}\left(x_{1}-\left(x-\frac{i a z}{x_{0} k}\right)\right)^{2}\right) A i\left(\frac{x_{1}}{x_{0}}\right) \times \\
& \left.\int d y_{1} \exp \left(\frac{i k}{2 z}\left[y_{1}-\left(y-\frac{i a z}{y_{0} k}\right)\right]^{2}\right) A i\left(\frac{y_{1}}{y_{0}}\right)\left(y_{1}-y_{d}\right)\right\}
\end{aligned}
$$

By utilizing the convolution theorem, we can write the field of the beam as follows [12].

$$
\begin{aligned}
& E(x, y, z)=\frac{i k}{2 \pi z} \frac{x_{0}}{\sqrt{2 \pi}} \frac{y_{0}}{\sqrt{2 \pi}}\left(\sqrt{\frac{-i z}{k}}\right)^{2} \exp \left(\frac{a x}{x_{0}}\right) \times \\
& \exp \left(\frac{a y}{y_{0}}\right) \int\left[\int-\frac{z \xi}{k} \exp \left(\frac{-i x_{0}^{3} \xi^{3}}{3}-\frac{i z \xi^{2}}{2 k}-i \xi x+\frac{a z \xi}{x_{0} k}\right) d \xi\right. \\
& \left.\int\left(x-\frac{i a z}{x_{0} k}-x_{d}\right) \exp \left(\frac{-i x_{0}^{3} \xi^{3}}{3}-\frac{i z \xi^{2}}{2 k}-i \xi x+\frac{a z \xi}{x_{0} k}\right) d \xi\right] \times \\
& \int \exp \left(\frac{-i y_{0}^{3} \xi^{3}}{3}-\frac{i z \xi^{2}}{2 k}-i \xi y+\frac{a z \xi}{y_{0} k}\right) d \xi+ \\
& i \int \exp \left(\frac{-i x_{0}^{3} \xi^{3}}{3}-\frac{i z \xi^{2}}{2 k}-i \xi x+\frac{a z \xi}{x_{0} k}\right) d \xi \times
\end{aligned}
$$

$$
\begin{aligned}
& {\left[\int \frac{z \xi}{k} \exp \left(\frac{-i y_{0}^{3} \xi^{3}}{3}-\frac{i z \xi^{2}}{2 k}-i \xi y+\frac{a z \xi}{y_{0} k}\right) d \xi+\right.} \\
& \left.\left\{\left(y-\frac{i a z}{y_{0} k}-y_{d}\right) \exp \left(\frac{-i y_{0}^{3} \xi^{3}}{3}-\frac{i z \xi^{2}}{2 k}-i \xi y+\frac{a z \xi}{y_{0} k}\right) d \xi\right]\right\}
\end{aligned}
$$

Equation (5) can be expressed as follows

$$
\begin{aligned}
& E(x, y, z)=\frac{x_{0} y_{0}}{(2 \pi)^{2}} \exp \left(\frac{a x}{x_{0}}+\frac{a y}{y_{0}}\right) \exp \left(\frac{-a z^{2}}{2 k^{2} x_{0}^{4}}-\frac{a z^{2}}{2 k^{2} y_{0}^{4}}\right) \times \\
& \exp \left(\frac{i z x}{2 k x_{0}^{3}}+\frac{i z y}{2 k y_{0}^{3}}+\frac{i z^{3}}{24 k^{3} x_{0}^{3}}+\frac{i z^{3}}{24 k^{3} y_{0}^{3}}-\frac{i z^{3}}{8 k^{3} x_{0}^{6}}-\frac{i z^{3}}{8 k^{3} y_{0}^{6}}\right) \times \\
& \left\{\left[\frac{i k}{z} A i^{\prime}\left(\frac{x}{x_{0}}-\frac{z^{2}}{4 k^{2} x_{0}^{4}}+\frac{i a z}{x_{0}^{2} k}\right)+\left(x-\frac{i a z}{x_{0} k}-x_{d}\right) \times\right.\right. \\
& \left.A i\left(\frac{x}{x_{0}}-\frac{z^{2}}{4 k^{2} x_{0}^{4}}+\frac{i a z}{x_{0}^{2} k}\right)\right] \times A i\left(\frac{y}{y_{0}}-\frac{z^{2}}{4 k^{2} y_{0}^{4}}+\frac{i a z}{y_{0}^{2} k}\right)+ \\
& i A i\left(\frac{x}{x_{0}}-\frac{z^{2}}{4 k^{2} x_{0}^{4}}+\frac{i a z}{x_{0}^{2} k}\right) \times\left[\frac{i k}{z} A i^{\prime}\left(\frac{y}{y_{0}}-\frac{z^{2}}{4 k^{2} y_{0}^{4}}+\frac{i a z}{y_{0}^{2} k}\right)+\right.
\end{aligned}
$$

$$
\left.\left.\left(y-\frac{i a z}{y_{0} k}-y_{d}\right) A i\left(\frac{y}{y_{0}}-\frac{z^{2}}{4 k^{2} y_{0}^{4}}+\frac{i a z}{y_{0}^{2} k}\right)\right]\right\}
$$

\section{B. Fractional FT}

Figure 1 is the general expression of the field of an $\mathrm{AiB}$ with OV passing through an optical system. The Lohmann I optical system and the Lohmann II optical system are equivalent, and are described by the following transfer matrix [14]:

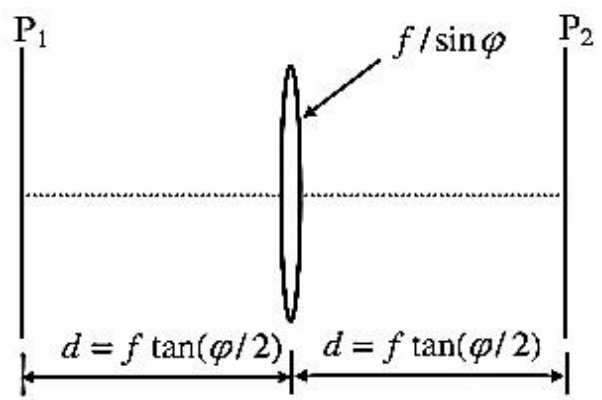

(a)

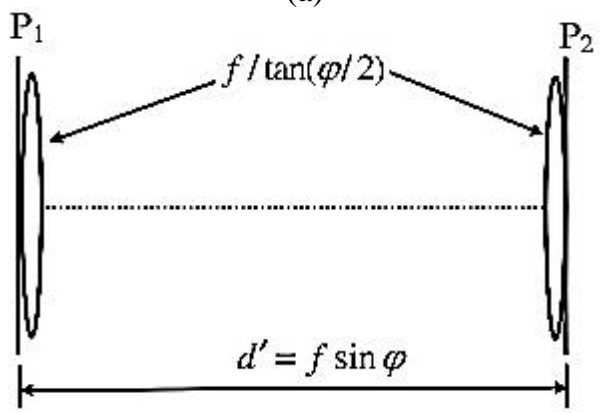

(b)

Fig. 1. Optical system for the FrFT, for Lohmann (a) I and (b) systems. 


$$
R=\left(\begin{array}{ll}
A & B \\
C & D
\end{array}\right)=\left(\begin{array}{cc}
\cos \varphi & f \sin \varphi \\
-1 / f \sin \varphi & \cos \varphi
\end{array}\right)
$$

where, $\varphi=\pi p / 2$ and $p$ is the order of fractional FT [14]. In Fig 1 (a), the distance between the input and output planes is $2 d=2 f \tan (\varphi / 2)$. In Fig 1 (b), the distance between the input and output planes is $d^{\prime}=f \sin (\varphi)$. Under the paraxial approximation, the Collin formula can be used to calculate the field of the AiB passing through the Lohmann optical system [12].

$$
\begin{aligned}
& E(x, y, z)=\frac{i}{\lambda B} \exp (i k z) \\
& \iint d x_{1} d y_{1}\left\{\operatorname { e x p } \left[\frac{i \pi}{\lambda B} A\left(x_{1}^{2}+y_{1}^{2}\right)-2\left(x x_{1}+y y_{1}\right)\right.\right. \\
& \left.\left.D\left(x^{2}+y^{2}\right)\right] E\left(x_{1}, y_{1}, z=0\right)\right\}
\end{aligned}
$$

Finally, the field of the AiB with OV under fractional FT after propagating a distance $z$ by employing the convolution theorem, can be formulated as:

$$
\begin{aligned}
& E(x, y, z)=\frac{\exp (i k z)}{i \lambda B} \exp \left[\frac{i k D\left(x^{2}+y^{2}\right)}{2 B}\right] \\
& \exp \left(\frac{a x}{x_{0}}+\frac{a y}{y_{0}}\right) \exp \left(\frac{-i k x^{2}}{2 B A}-\frac{i k y^{2}}{2 B A}+\frac{i a^{2} B}{2 A k x_{0}^{2}}\right) \times \\
& \left\{\left[\int _ { - \infty } ^ { \infty } d \xi ( \frac { - B \xi } { k A } + \frac { x } { A } + \frac { i a B } { k A x _ { 0 } } - x _ { d } ) \operatorname { e x p } \left(-\frac{i x_{0}^{3} \xi^{3}}{3}-\right.\right.\right. \\
& \left.\frac{i B \xi^{2}}{2 k A}-i \xi\left(\frac{x}{A}+\frac{i a B}{k A x_{0}}\right)\right) \int_{-\infty}^{\infty} d \xi \exp \left[\left(-\frac{i y_{0}^{3} \xi^{3}}{3}-\right.\right. \\
& \left.\frac{i B \xi^{2}}{2 k A}-i \xi\left(\frac{y}{A}+\frac{i a B}{k A y_{0}}\right)\right]+\left[i \int _ { - \infty } ^ { \infty } d \xi \operatorname { e x p } \left[\left(-\frac{i x_{0}^{3} \xi^{3}}{3}-\right.\right.\right. \\
& \left.\frac{i B \xi^{2}}{2 k A}-i \xi\left(\frac{x}{A}+\frac{i a B}{k A x_{0}}\right)\right] \int_{-\infty}^{\infty} d \xi\left(\frac{-B \xi}{k A}+\frac{y}{A}+\frac{i a B}{k A y_{0}}-\right. \\
& \left.\left.y_{d}\right) \exp \left[-\frac{i y_{0}^{3} \xi^{3}}{3}-\frac{i B \xi^{2}}{2 k A}-i \xi\left(\frac{y}{A}+\frac{i a B}{k A y_{0}}\right)\right]\right\}
\end{aligned}
$$

Eq. (8) can be expressed as follows

$$
\begin{aligned}
& E(x, y, z)=\frac{\exp (i k z)}{i \lambda B} \exp \left[\frac{i k D\left(x^{2}+y^{2}\right)}{2 B}\right] \exp \left(\frac{a x}{x_{0}}+\right. \\
& \left.\frac{a y}{y_{0}}+\frac{a B^{2}}{2 k^{2} A x_{0}^{4}}+\frac{a B^{2}}{2 k^{2} A y_{0}^{4}}\right) \exp \left(\frac{-i k x^{2}}{2 B A}-\frac{i k y^{2}}{2 B A}+\right.
\end{aligned}
$$

$$
\begin{aligned}
& \left.\frac{i a^{2} B}{2 A k x_{0}^{2}}+\frac{i a^{2} B}{2 A k x_{0}^{2}}\right) \exp \left(\frac{i B^{3}}{24 k^{3} x_{0}^{3} A^{3}}+\frac{i B^{3}}{24 k^{3} y_{0}^{3} A^{3}}-\right. \\
& \left.\frac{i B^{3}}{8 k^{3} x_{0}^{4} A^{3}}-\frac{i B^{3}}{8 k^{3} y_{0}^{4} A^{3}}+\frac{i x B}{2 k A^{2} x_{0}^{3}}+\frac{i y B}{2 k A^{2} y_{0}^{3}}\right)\left\{\left(\left[\frac{i k}{B} \times\right.\right.\right. \\
& A i^{\prime}\left(\frac{x}{x_{0} A}-\frac{B^{2}}{4 k^{2} A^{2} x_{0}^{2}}+\frac{i a B}{k A x_{0}^{2}}\right)+\left(\frac{x}{A}-x_{d}+\frac{i a B}{k A x_{0}}\right) \times \\
& \left.A i\left(\frac{x}{x_{0} A}-\frac{B^{2}}{4 k^{2} A^{2} x_{0}^{2}}+\frac{i a B}{k A x_{0}^{2}}\right)\right] \times A i\left(\frac{y}{y_{0} A}-\frac{B^{2}}{4 k^{2} A^{2} y_{0}^{2}}+(9)\right. \\
& \left.\left.\frac{i a B}{k A y_{0}^{2}}\right)\right)+i A i\left(\frac{x}{x_{0} A}-\frac{B^{2}}{4 k^{2} A^{2} x_{0}^{2}}+\frac{i a B}{k A x_{0}^{2}}\right) \times \\
& {\left[A i^{\prime}\left(\frac{y}{y_{0} A}-\frac{B^{2}}{4 k^{2} A^{2} y_{0}^{2}}+\frac{i a B}{k A y_{0}^{2}}\right)+\left(\frac{y}{A}-y_{d}+\frac{i a B}{k A y_{0}}\right) \times\right.} \\
& \left.\left.A i\left(\frac{y}{y_{0} A}-\frac{B^{2}}{4 k^{2} A^{2} y_{0}^{2}}+\frac{i a B}{k A y_{0}^{2}}\right)\right]\right\}
\end{aligned}
$$

\section{NUMERICAL SimUlationS AND ANALYSIS}

It was assumed that $\lambda=0.53 \mu \mathrm{m}, f=1000 \mathrm{~mm}$, $x_{0}=y_{0}=0.1 \mathrm{~mm}, x_{d}=y_{d}=0.3 \mathrm{~mm}$ and $a=10^{-5}$. In this simulation, Eq. (5), Eq. (9), paraxial approach and the method of the Wigner distribution function were used. Fig. 2 shows the twodimensional intensity pattern for AiB. From Fig. 2(a) it can be found that the uniformity of the lateral lob of the beam with OV disappears while, in Fig. 2(b), the peak uniformity of the beam without $\mathrm{OV}$ increases. It can also be seen in Fig. 2 (a), that the effective size of the beam is decreased, the main peak is straitened and the width and height of the other peaks are also decreased.

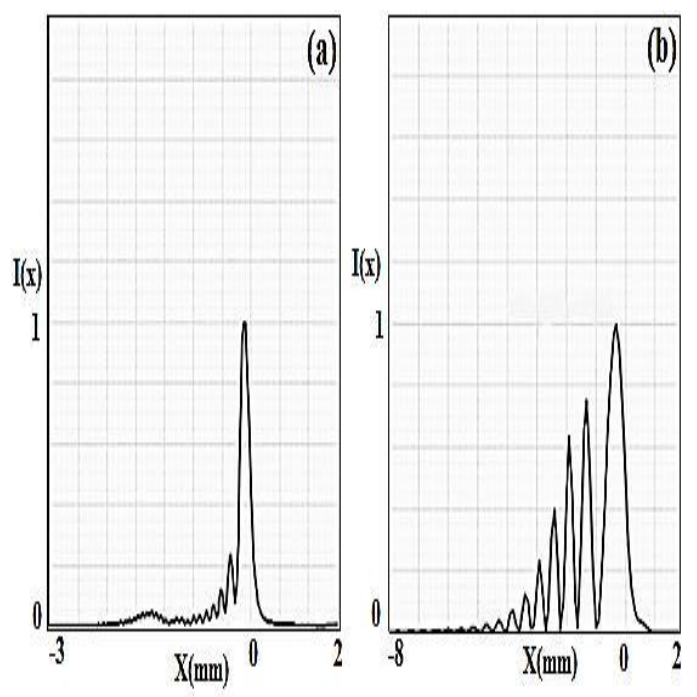



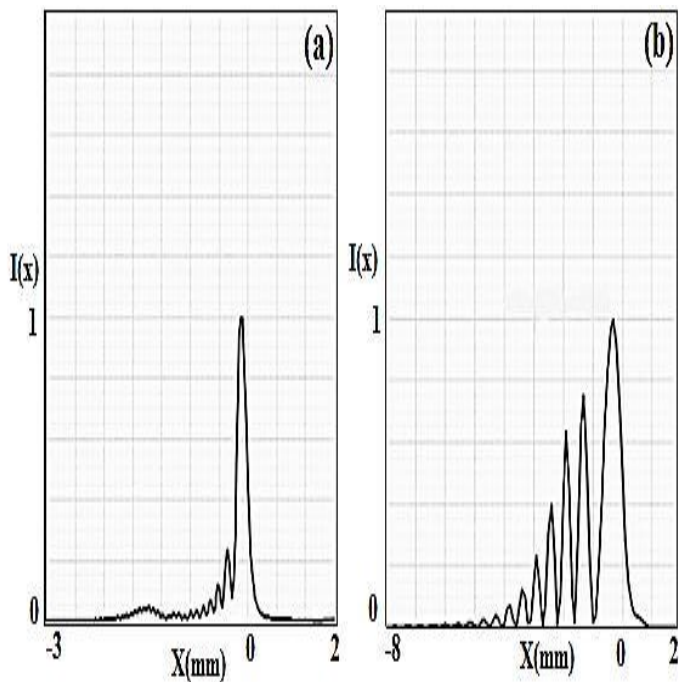

Fig. 2. Intensity normalized in the $x$ - direction of $\mathrm{AiB}$; (a) with OV; (b) without OV.

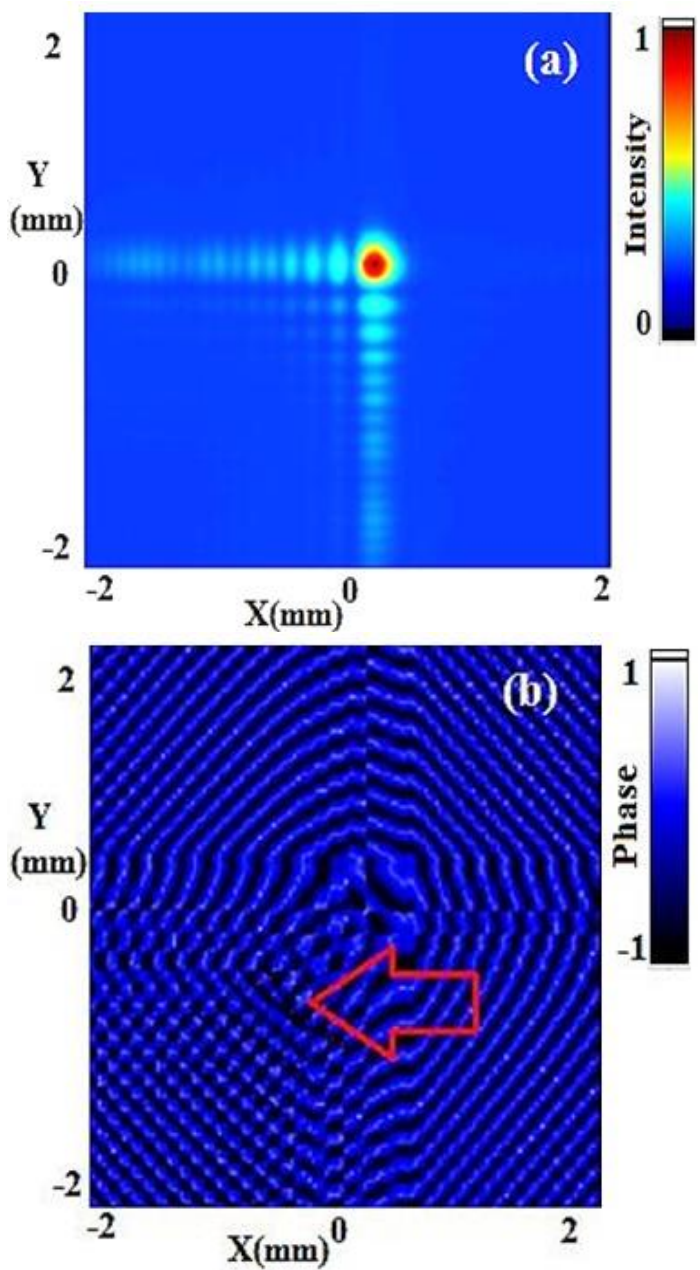

Fig. 3. AiB with OV; (a) intensity and (b) phase pattern.
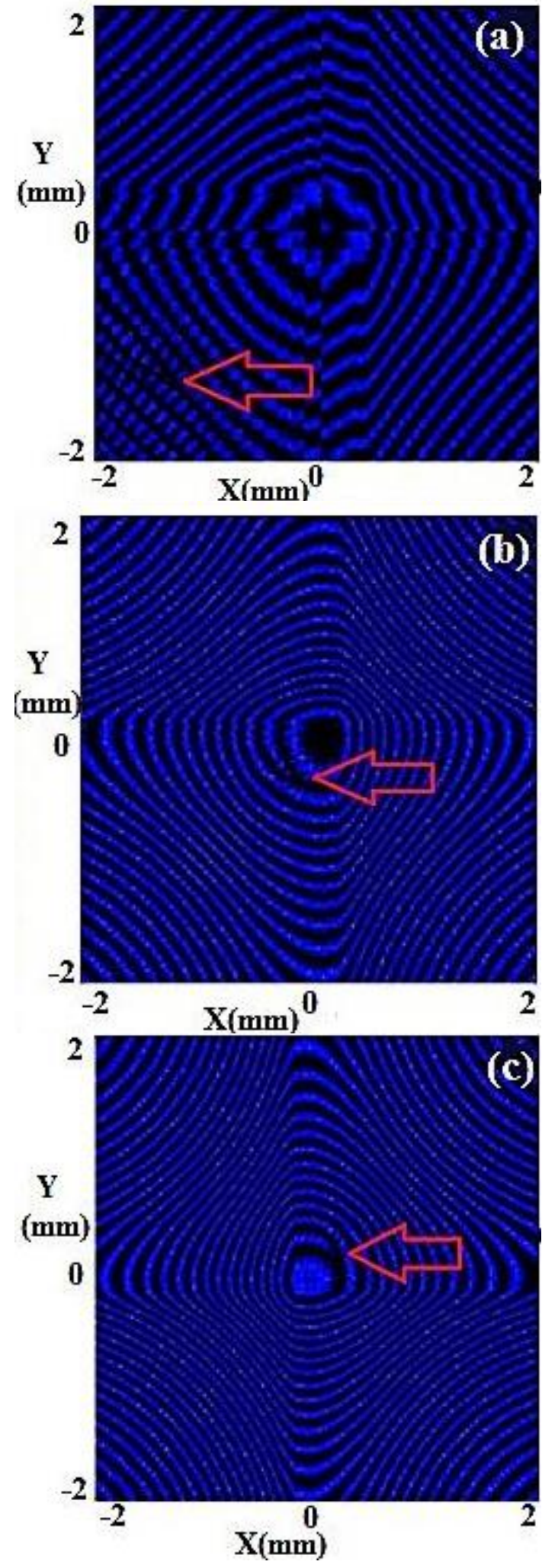


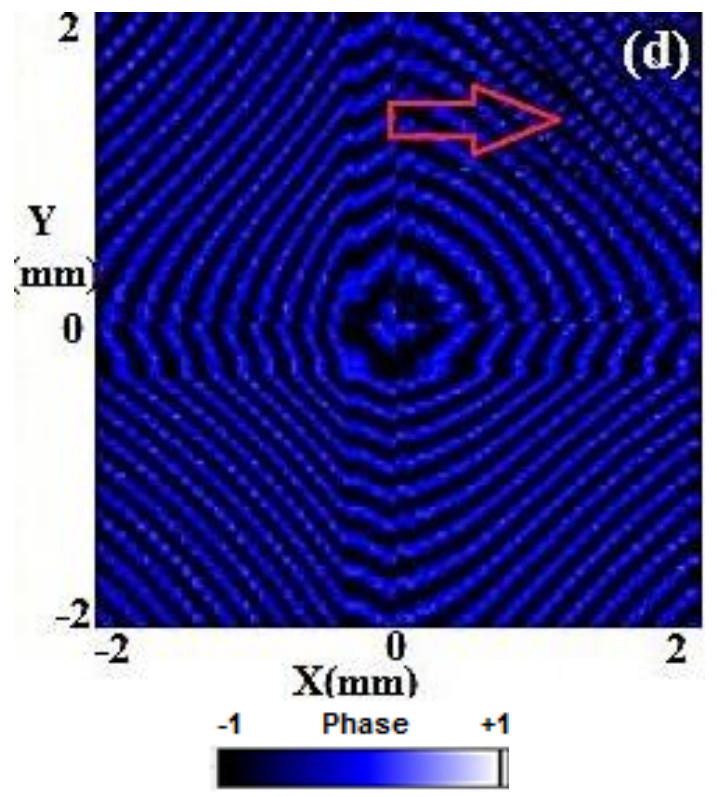

Fig. 4. Phase pattern of the AiB with OV for; the power of fractional FT for (a) $p=0.3$, (b) $p=0.7$, (c) $p=1.3$, (d) $p=1.7$.

Fig. 3 shows the intensity pattern (a) and phase pattern (b). In Fig. 3 (b), the arrow represents the position of the OV. Fig. 4 shows the phase and intensity patterns of an $\mathrm{AiB}$ with $\mathrm{OV}$ for the different powers of fractional FT, the direction of the patterns changes, which is a logical conclusion of Eq. (7).

The position of the OV changes with increasing $p$, and this can be seen in Fig. 4 (a). If the value of $p$ is closer to 1 , the effective beam size will decrease. In Fig. 4 (b), with approaching $p$ to 1 , the main peak straitens.

In Fig. 5, for $p<1$, the beam spot of the AiB decreases when the value of $p$ is increased. For $1<p<2$, the beam spot of the AiB increases with increasing the value of $p$. Fig. 6 shows the two-dimensional intensity graphs for different $p$. In this case, if the value of $p$ is closer to 1 the lateral side lobs will be far from the $x$ axis.
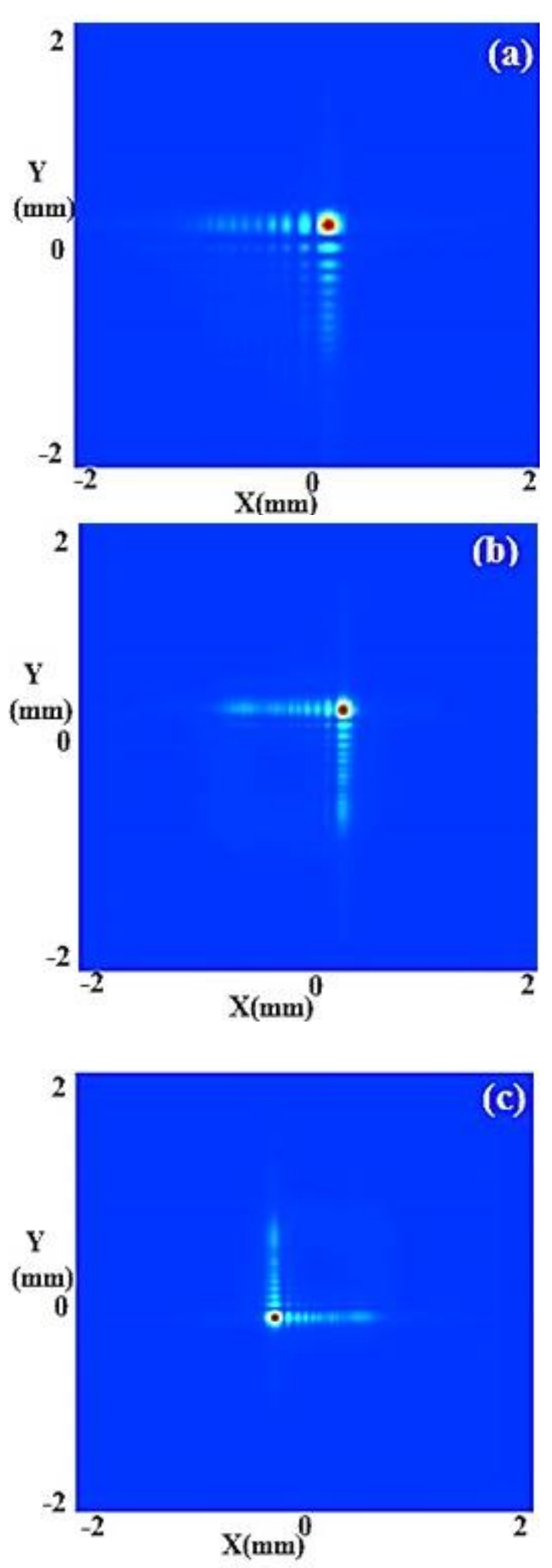


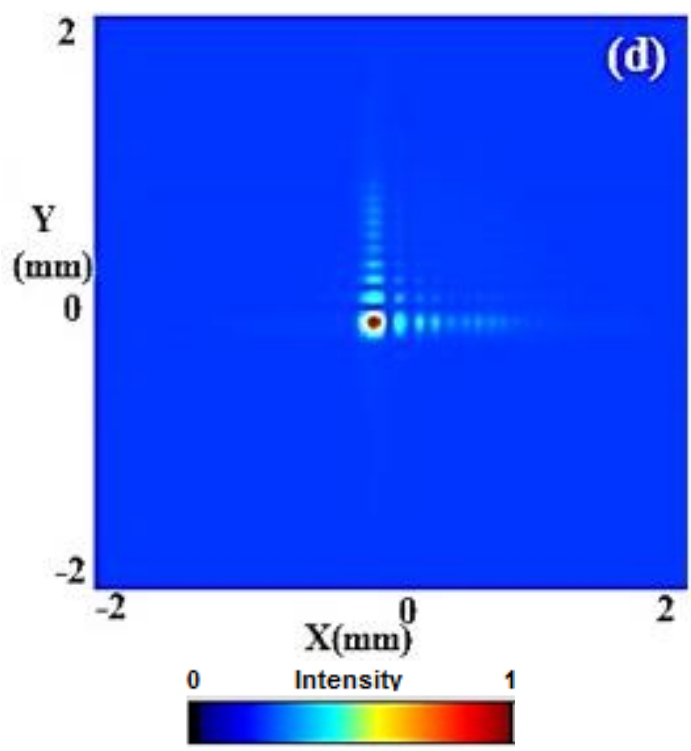

Fig. 5. Intensity pattern of the $\mathrm{AiB}$ with $\mathrm{OV}$ for; the power of fractional FT for (a) $p=0.3$, (b) $p=0.7$, (c) $p=1.3$, (d) $p=1.7$.
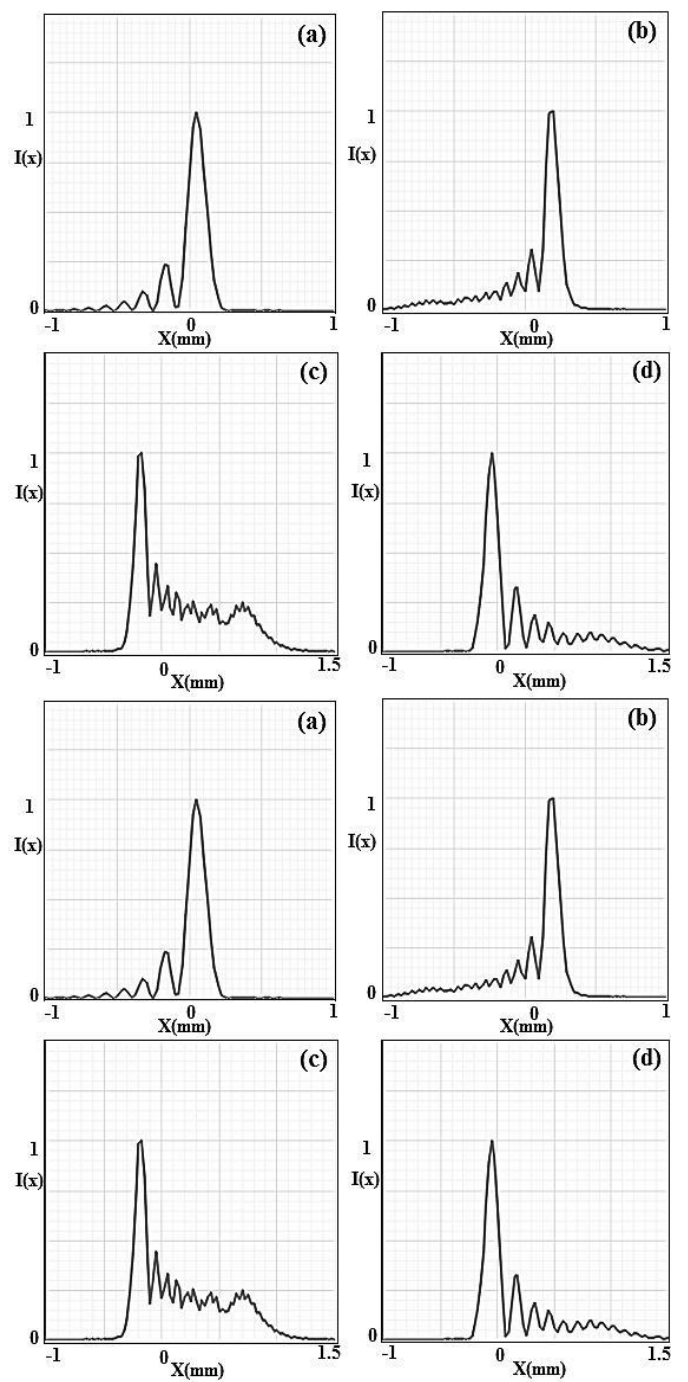
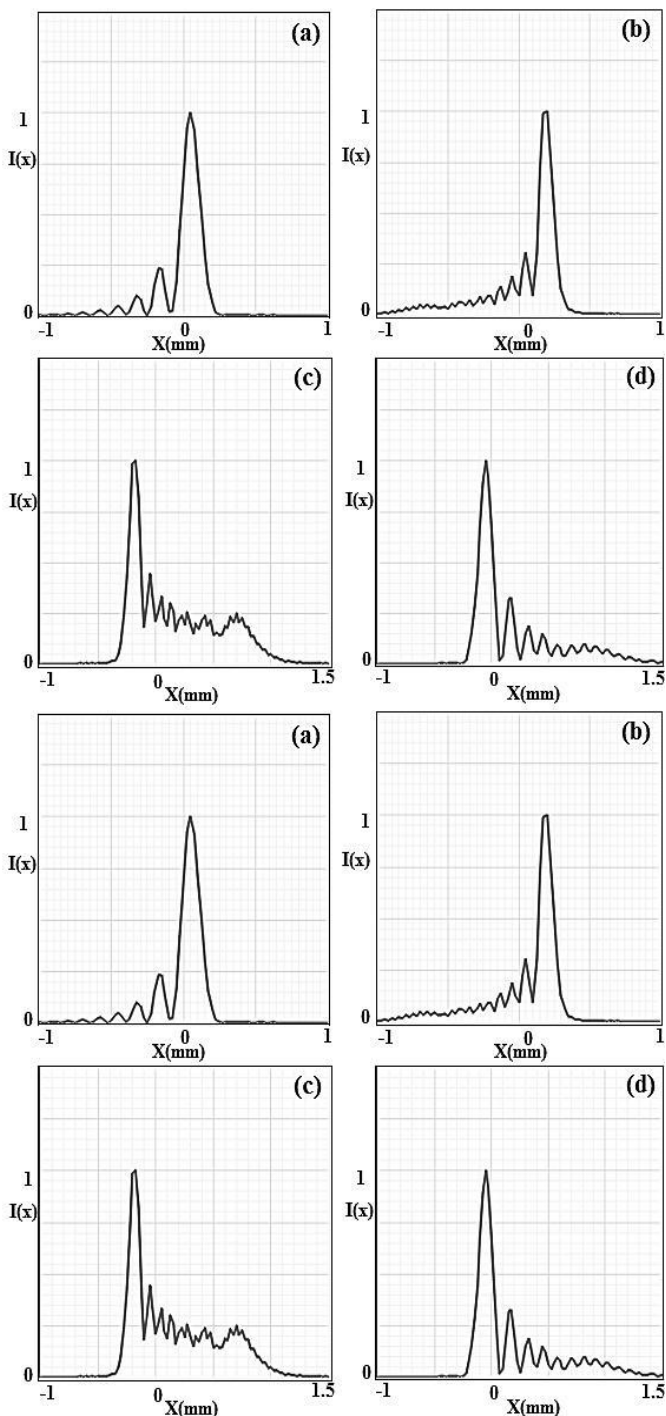

Fig. 6. Intensity normalized in the $x$ - direction of $\mathrm{AiB}$ with $\mathrm{OV}$; (a) $p=0.3$, (b) $p=0.7$, (c) $p=1.3$, (d) $p=1.7$.

Figure 7 shows the two-dimensional spot beam size $(\mathrm{S})$ graph of the $\mathrm{AiB}$ with $\mathrm{OV}$ for different $p$. for $p<1$, the beam spot of the AiB decreases when the value of $p$ is increased. For $1<p<2$, the beam spot of the AiB increases with increasing value of $p$. Figs. 8 and 9 show the phase and intensity patterns for an AiB with $\mathrm{OV}$. We consider the propagation of an $\mathrm{AiB}$ with $\mathrm{OV}$ and the propagation of an $\mathrm{AiB}$ without $\mathrm{OV}$ for fixed parameters $\lambda, f, a, x_{d}, x_{0}$ and by varying the value of $z$. In this case for each $\mathrm{z}$, there is a unique transfer matrix, which depends on the distance between the two primary and final pages. 


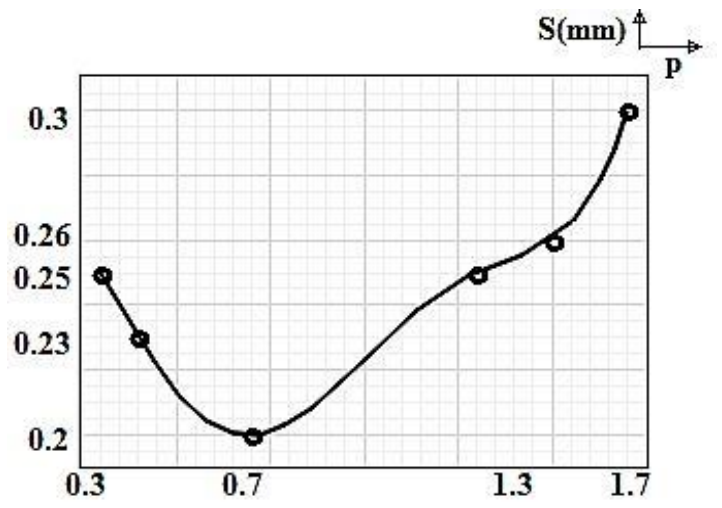

Fig. 7. Spot beam size $(S)$ of the $\mathrm{AiB}$ with $\mathrm{OV}$ for the powers of the fractional FT $(p)$.

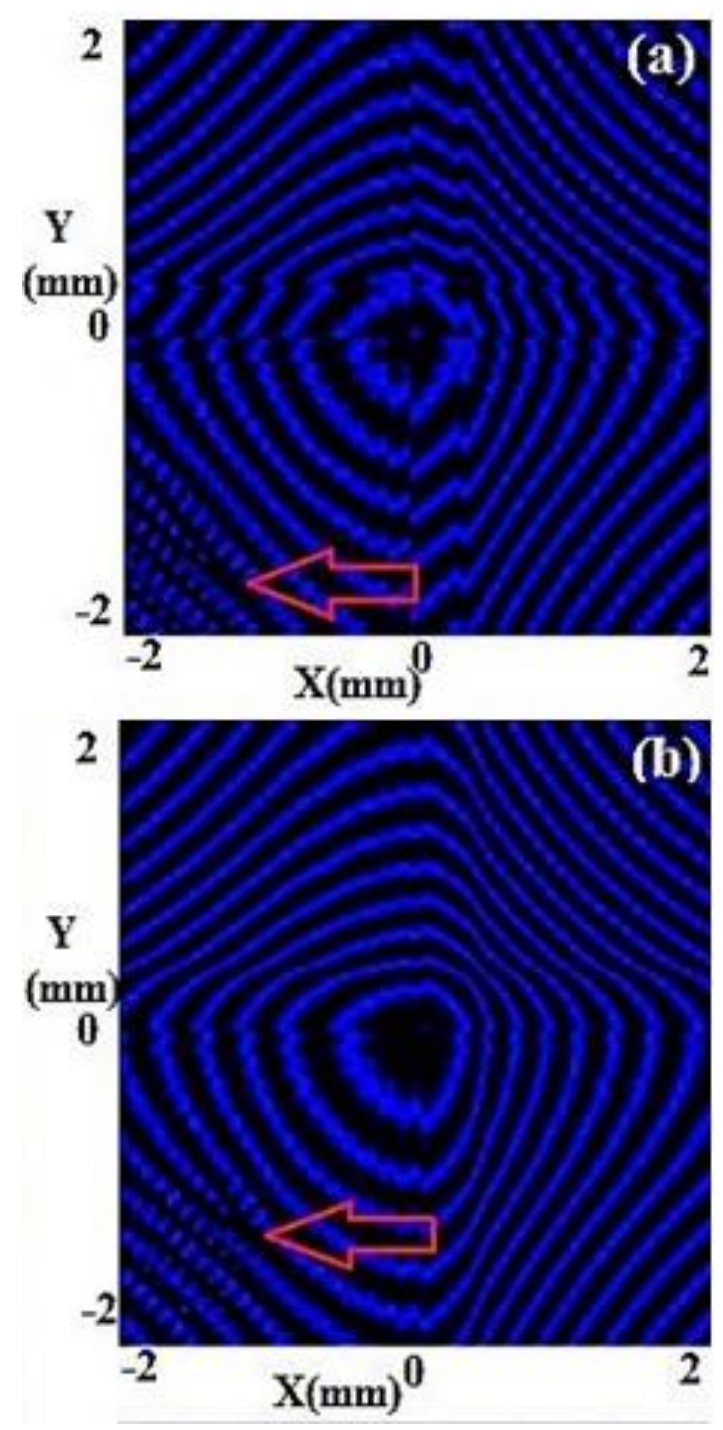

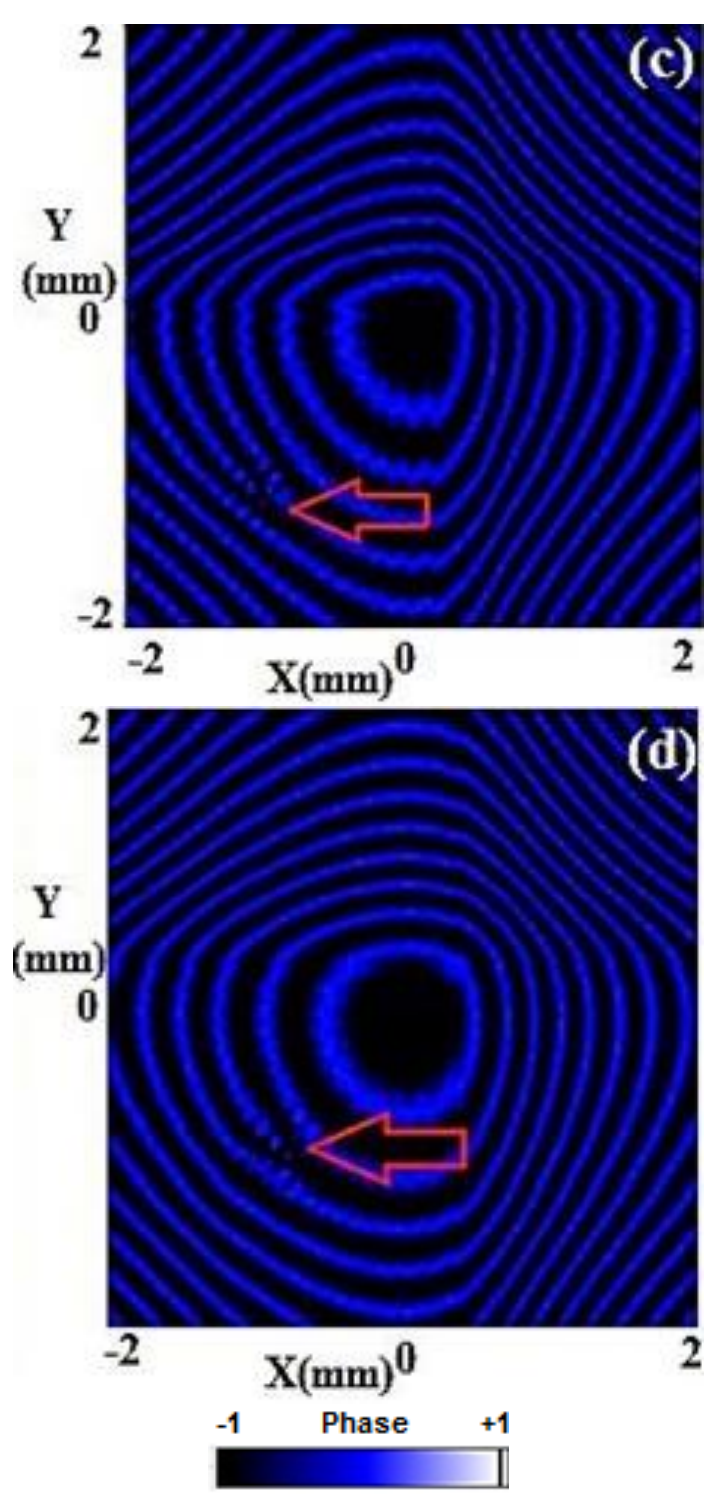

Fig. 8. Phase pattern of the AiB with OV for $p=0.5$ at; (a) $z=0.4 d$; (b) $z=0.5 d$, (c) $z=0.6 d$, (d) $z=0.8 d$.

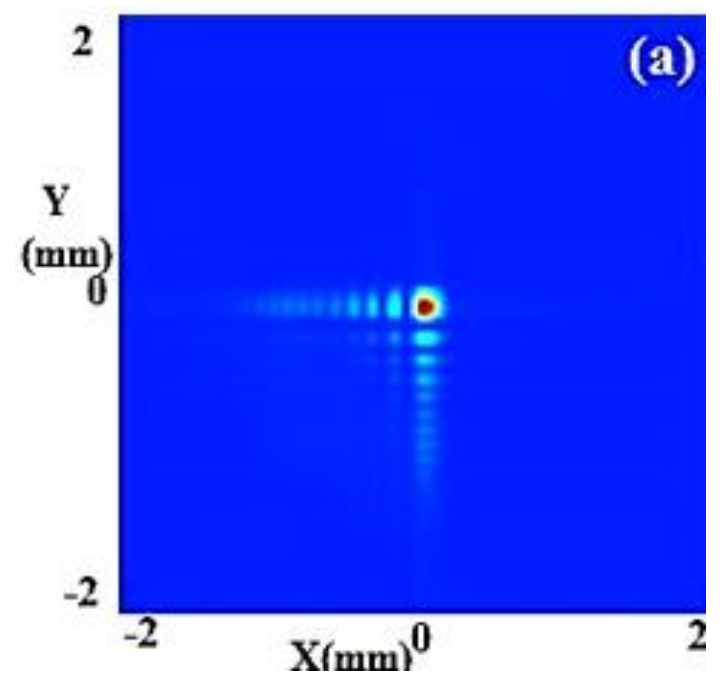



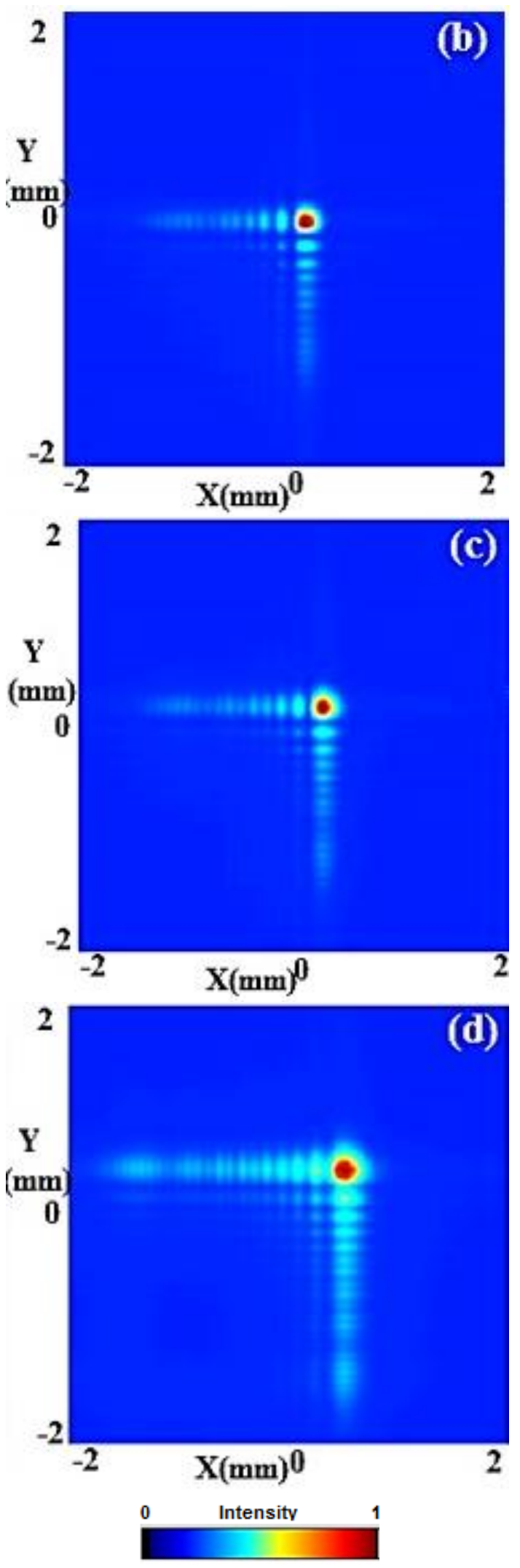

Fig. 9. Intensity pattern of the $\mathrm{AiB}$ with $\mathrm{OV}$ for $p=0.5$ at; (a) $z=0.4 d$; (b) $z=0.5 d$, (c) $z=0.6 d$, (d) $z=0.8 d$.
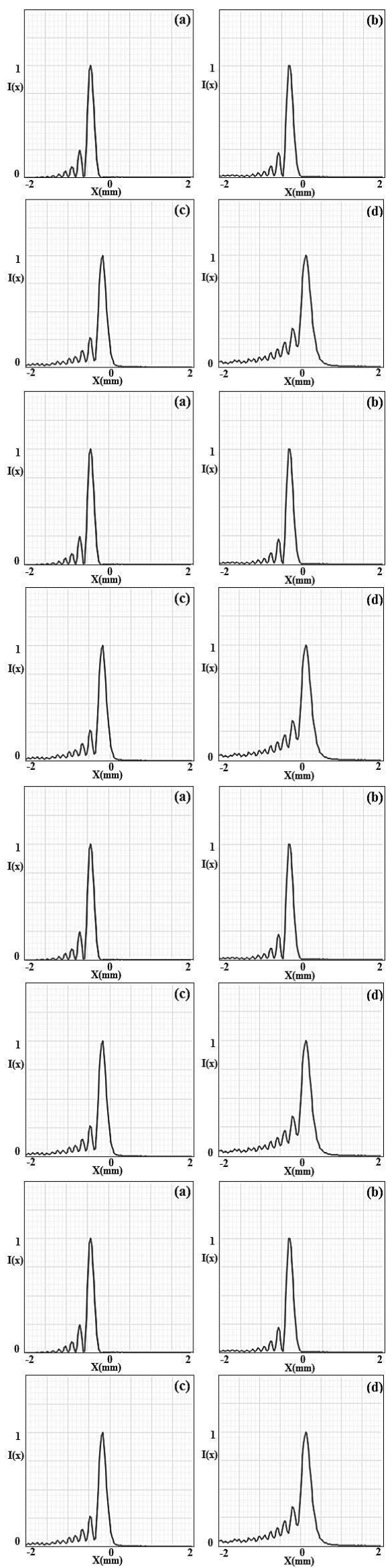

Fig. 10. Intensity normalized in the $x$ - direction of $\mathrm{AiB}$ with OV for $p=0.5$ at; (a) $z=0.4 d$; (b) $z=0.5 d$, (c) $z=0.6 d$, (d) $z=0.8 d$. 
Figure 10 shows the two-dimensional intensity graphs of Eq. (8) for different $z$. In Fig. 10, the lateral side lobs will be far from the $x$ axis when the value of $z$ increases.

Figure 11 shows the two-dimensional spot beam size $(\mathrm{S})$ graph of the $\mathrm{AiB}$ with $\mathrm{OV}$ for different $\mathrm{z}$. With increasing the value of $\mathrm{z}$, the main peak expands.

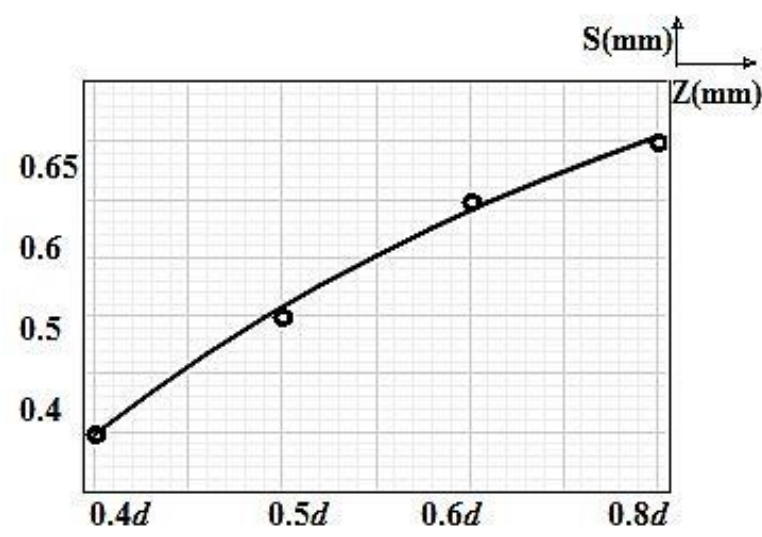

Fig. 11. Spot beam size $(S)$ of the AiB with $\mathrm{OV}$ for different distance $(Z)$.

\section{CONCLUSION}

The new results and the results obtained earlier revealed that, the order of fractional FT $p$ not only affects the beam spot size of an $\mathrm{AiB}$, but also controls the orientation of the beam spot. The position of the OV in an optical system changes with changing the power of the fractional FT $(p)$. In phase pattern, the process of $p$ from 0 to 1 , the position of the $\mathrm{OV}$ is closer to the center. Also, when the power of the fractional FT $(p)$ is changed, the patterns of intensity and phase also change. In the intensity pattern of $\mathrm{AiB}$, there is a uniform increase in the height of the peaks. Lobs of the $\mathrm{AiB}$ with $\mathrm{OV}$ are destroyed. With $p$ tending towards 1, the main peak straitens and the number of spots increases. With increasing the value of $z$, the main peak expands and the effective beam size increases. If the value of $p$ is closer to 1 and the value of $z$ increases, the lateral side lobs will be far from the $x$ axis. When $p<1$, the beam spot of the AiB decreases with increasing value of $p$, and when $1<p<2$ the beam spot of the AiB increases with increasing value of $p$. It was concluded that the behavior of the $\mathrm{AiB}$ with $\mathrm{OV}$ controls changes the order of the fractional FT in the optical system.

\section{REFERENCES}

[1] M.V. Berry and N.L. Balazs, "Non spreading wave packets," Am. J. Phys. Vol. 47, pp. 264268, 1979.

[2] G.A. Siviloglou, J. Broky, A. Dogariu, and D. N. Christodoulides, "Observation of accelerating AiBs," Phys. Rev. Lett. Vol. 99, pp. 213901-213913, 2007.

[3] G.A. Siviloglou and D.N Christodoulides, "Accelerating finite energy AiBs," Opt. Lett. Vol. 32, pp. 9411-9423, 2008.

[4] H.I. Sztul and R. Alfano, "Double-slit interference with Laguerre-Gaussian beams," Opt. Express, Vol. 16, pp. 999-1001, 2008.

[5] R. Chen, H. Zheng, and C. Dai, "Wigner distribution function of an AiB," Opt. Soc. Am. A, Vol. 28, pp. 1307-1322, 2011.

[6] P. Polynkin and M. Kolesik, "Nonlinear propagation dynamics of finite-energy AiBs," Phys. Rev. Lett. Vol. 103, pp. 123902-123966, 2009.

[7] Y. Gu and G. Gbur, "Scintillation of AiB arrays in atmospheric turbulence," Opt. Lett. Vol. 35, pp. 3456-3458, 2010.

[8] W. Wen, X. Lu, Ch. Zhao, and Y. Cai, "Propagation of $\mathrm{AiB}$ passing through the misaligned optical system with hard aperture," Optic. Commun. Vol. 313, pp. 350-355, 2014.

[9] H.T. Dai, Y.J. Liu, D. Luo, and X.W. Sun, "Propagation dynamics of an OV imposed on an AiB," Opt. Lett. Vol. 35, pp. 4075-4077, 2010.

[10] W. Wen and X. Chu, "Beam wander of an AiB with a spiral phase," Opt. Soc. Am. A, Vol. 31, pp. 5202-5204, 2014.

[11]S. Hua, Y. Liu, H. Zhang, L. Tang, and Y. Feng, "Propagation of an Airy-Gaussian-OV beam in chiral medium," Opt. Commun, Vol. 388, pp. 29-33, 2017.

[12]G. Zhou, R. Chen, and X. Chu, "Fractional Fourier transform of AiBs," Appl. Phys. B, Vol. 109, pp. 549-556, 2012.

[13]S.M. Azmayesh-Fard, "Gaussian beam propagation," Opt. Soc. Am. A, Vol. 30, pp 640-652, 2013. 
[14]A.W. Lohmann, "Image rotation, Wigner rotation, and the fractional Fourier transform," Opt. Soc. Am. A, Vol. 10, pp. 2181-2186, 1993.

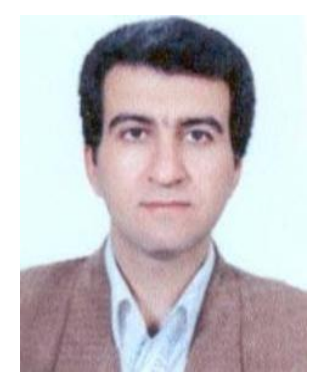

Mohammad Moradi was born in 1965. He received the B.Sc. degree in Physics in 1989 from Isfahan University, Isfahan, Iran; M.Sc. degree in Atomic Physics in 1992 from Polytechnic Tehran University, Iran, and Ph.D. degree in Atomic Physics, Optics and Laser from Moscow State University in 2005, Russia. He is currently engaged as an Assistant
Professor of Physics in Department of Physics, Shahrekord University, Shahrekord, Iran.

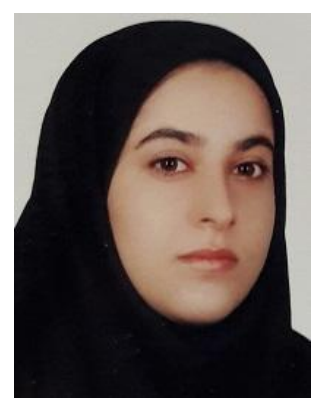

Forouzan Habibi was born in 1990. She received her B.Sc. degree and M.Sc. degree in Atomic and Molecular Physics from Shahrekord University, Shahrekord, Iran in 2012 and 2017, respectively. She is currently a $\mathrm{PhD}$ candidate of Physics (Optics and laser) in Mazandaran University, Iran. 